\title{
An introduction to clinical governance in dentistry
}

\author{
Kishan Patel ${ }^{* 1}$ and lan Jenkyn ${ }^{2}$
}

\section{Key points}

\begin{abstract}
Clinical governance permeates all aspects of dental practice regardless of speciality or workplace. Many of the principles of clinical governance form part of essential legal and professional obligations for continued practice as a dentist. In light of this, this article aims to highlight the core concepts of clinical governance and aid dental practitioners to apply these to patient care.
\end{abstract}

\section{Introduction}

Clinical governance is fundamentally important to our daily clinical practice as dentists, with its concepts traversing primary, secondary and tertiary medical and dental care. It is applicable to both NHS and private practice and is mandated by the Care Quality Commission stating 'all healthcare providers should give clinical governance a high priority.' The NHS has defined clinical governance as: 'A framework through which NHS organisations are accountable for continuously improving the quality of their services and safeguarding high standards of care by creating an environment in which clinical care will flourish. ${ }^{2}$

Practising aspects of clinical governance is required by all members of staff within a healthcare setting, extending to both clinical and non-clinical members of the team. However, providing guidance, leadership and stewardship in these aspects falls to senior, designated members of the dental team. Examples of such individuals could be department audit leads in secondary care and practice managers or

${ }^{1}$ Specialist Registrar in Orthodontics, Educational Supervisor in LKSS Deanery, UK; ${ }^{2}$ Junior Clinical Fellow, Oral and Maxillofacial Surgery, Cambridge University

Hospitals, UK.

${ }^{*}$ Correspondence to: Kishan Patel

Email address: Kishan.patel@nhs.net

Refereed Paper.

Accepted 31 August 2020

https://doi.org/10.1038/s41415-021-2839-9 principal dentists in primary care. These roles are crucial to ensure compliance with extraclinical aspects of clinical governance are met.

Unfortunately, when providing clinical care for patients, mistakes are inevitable; it's the human nature of the service we provide. ${ }^{3}$ However, with a clear understanding of the remit of clinical governance, we can reduce such incidents and, where they do occur, we can learn from them to future-proof the delivery of care we provide.

This article will reintroduce and reinforce the different concepts of clinical governance. It will provide a practical understanding of the various pillars that comprise it and provide methods in which the dental practitioner can enhance their delivery of patient care to optimise clinical outcomes.

\section{Pillars of clinical governance}

The term clinical governance is an umbrella term encompassing various categories, or pillars. There aren't a set number of these categories, but the authors believe that all components of clinical governance can be divided into seven fluid areas:

1. Evidence-based care and effectiveness

2. Clinical audit

3. Risk management

4. Education, training and continuous professional development (CPD)

5. Patient and public involvement

6. Information management

7. Staff and staff management.
Some aspects of clinical governance, such as providing evidence-based care and practising clinical audit, can be implemented on both an individual and institutional level. Others, such as public involvement and staff management, may be slightly more difficult to implement for the average dental practitioner. Despite this, all pillars must be understood by all members of the team to ensure effective delivery. Each pillar will be discussed in more depth below.

\section{Evidence-based care and effectiveness}

Evidence-based care and effectiveness factors translate best evidence into decisions regarding the care of our patients. Evidencebased dentistry has been defined by the American Dental Association as 'an approach to oral health care that required the judicious integration of systematic assessments of clinically relevant scientific evidence, relating to the patient's oral and medical condition and history, with the dentist's clinical expertise and the patient's treatment needs and preferences. ${ }^{4}$ It is important that we amalgamate patients' values, clinical expertise and best possible evidence to improve our patient outcomes.

On an individual level, this can be actioned via practising critical appraisal, ${ }^{5}$ to ensure that the evidence is appropriately conducted and is relevant to your patient base. Adhering to established clinical guidelines is also 
another method of conducting this. At an organisational level, this can be monitored by measuring institutional practice to guidelines where relevant. An example of this could be to ascertain how often the Management of acute dental problems during COVID-19 pandemic ${ }^{6}$ guidance document was used by a dental practice during the UK-wide lockdown in the former half of 2020 .

\section{Clinical audit}

Clinical audit is a local, cyclical process whereby current practice is compared to set standards with the ultimate aim of improving the clinical care delivered. If practices don't meet standards, interventions are implemented and a re-audit carried out to determine if the interventions have improved practice such that they now meet standards (Fig. 1). Standards can either be set locally, established by the literature, or form part of national guidance. The first cycle of the audit can sometimes be thought of as 'quality assurance' as your audit may reveal set standards are already being met.

An example of nationally set audit guidance surrounds dental radiography. Public Health England and the Faculty of General Dental Practice (UK) have established guidance that no less than $70 \%$ of radiographs should be categorised as grade 1 and no more than $10 \%$ should be categorised as grade $3 .^{7}$ Practitioners and institutions alike can use these standards to measure their current practice to evaluate whether or not their practice meets this. If not, then appropriate interventions should be implemented and a re-audit undertaken.

It is important that all members of the dental team are involved in the audit process as it is not solely the responsibility of dentists. For example, a nurse may lead a cross-infection audit while audits relating to clinical procedures (such as radiology) should be undertaken by an appropriately trained member of the team, who would normally be the dentist.

\section{Risk management}

The inherent nature of providing dental care to our patients is risky. Risk fulfils the notion that an adverse patient incident (AI) may occur. It is important to understand that we cannot eliminate risk altogether within dentistry, but we can mitigate it with correct processes and procedures. Ultimately, risk management seeks to address the following points:

Fig. 1 A schematic diagram of an audit cycle

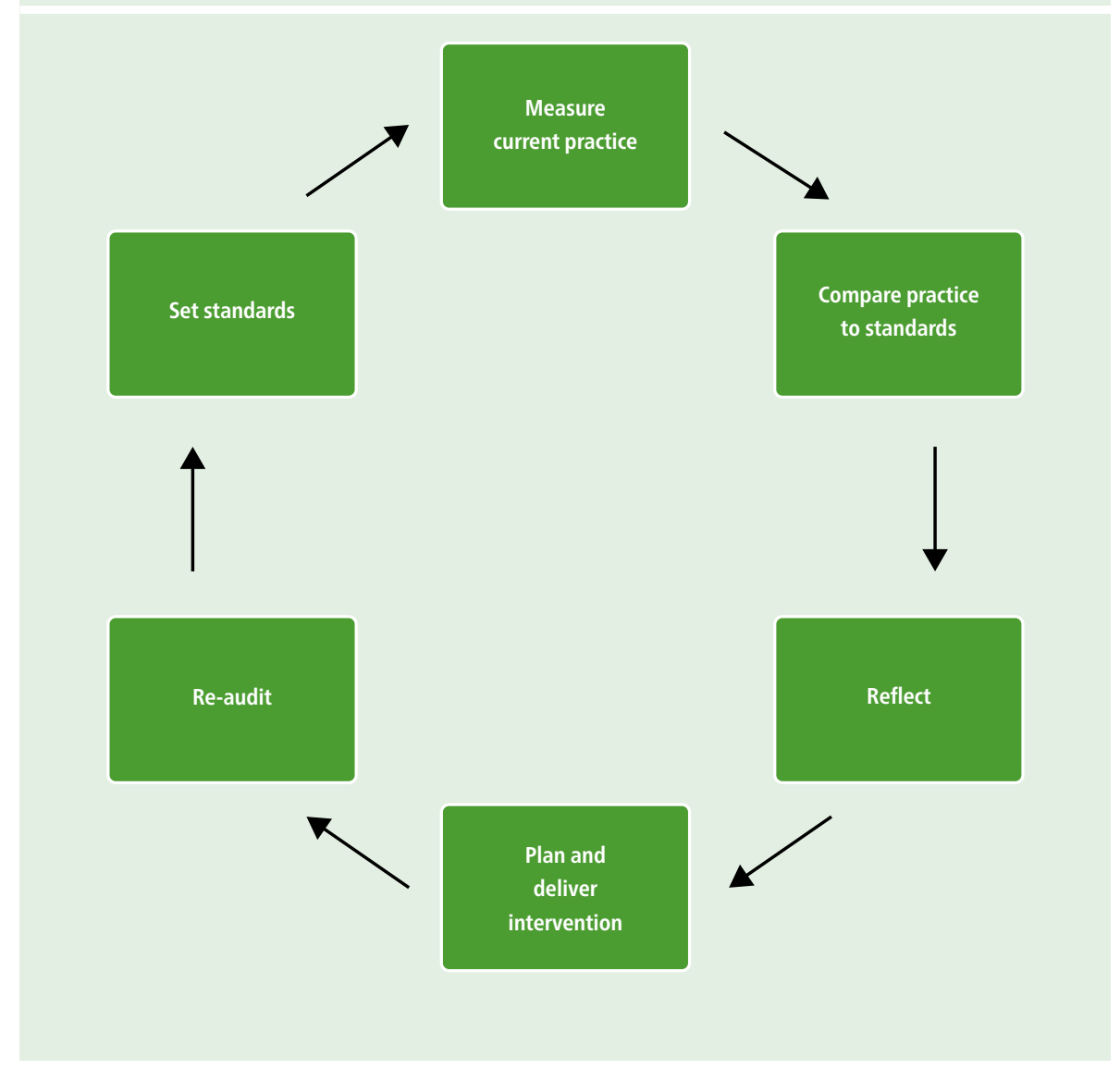

- Identify what can go wrong within an environment during patient care

- Understand the factors that can influence this

- Create a culture of openly reporting AIs, with a blame-free attitude

- Accept AIs will occur and to promote learning from such events

- Have systems in place to reduce risk and thereby reduce AIs.

An example of a process in place to reduce the risk of a wrong-site surgery, or 'never-event', is the World Health Organisation surgical safety checklist. ${ }^{8}$ It improves team communication before surgical procedures and has reduced the incidence of AIs globally. This is an example of an active step to reduce AIs within the healthcare setting.

However, in the instance that an AI does occur, it is important that the patient is informed and it is reported in the appropriate manner to allow analysis and learning. Duty of candour should be observed with all AIs. This is governed by Regulation 20 of the Health and Social Care Act 2008 (Regulated Activities) Regulations 2014. ${ }^{9}$ Duty of candour concerns promoting openness and transparency at all levels, from organisational to individual. Processes should be in place to ensure that this is observed, which can be implemented via appropriate staff training and awareness of official channels for AI reporting.

There are many methods of reporting AIs. Within a dental practice setting, this may be through scheduled practice meetings via a presentation highlighting the sequence of events that led to the AI. Within secondary care settings, there are 'Datix' forms which should be filled out for every AI. This creates an official log of events surrounding the AI which can be called on for future reference. This database can then be used to direct change of practice in areas identified as high risk for AIs in order to improve patient outcomes. Professional associations also offer modes of reporting AIs. For example, the British Orthodontic Society has an Adverse Incident Reporting page, allowing data to be collated and disseminated to the wider orthodontic community. ${ }^{10}$ In this way, the entire speciality can benefit, even if AIs are relatively rare.

Once an AI has been reported, it is important that the events leading up to the AI are analysed to inform checks and processes to reduce the risk for the future. ${ }^{11}$ This is also known as a 
root cause analysis (RCA). It is important that both human and system errors are identified. ${ }^{12}$ There are many ways in which this can be done. One example is using the concept of 'Five whys. ${ }^{13}$ This method involves constantly asking the question 'why' to any problem which was highlighted (Box 1). Another method to perform an RCA is using a fishbone diagram. ${ }^{14}$ This is a type of cause-and-effect analysis that allows the user to get to the base of the issue. Figure 2 provides an example from the NHS Improvement document regarding increased waiting times in a hospital setting. A simple brainstorming exercise with key stakeholders associated with the AI can also be held to determine causes for AIs. ${ }^{15}$

It is important that once an RCA has been undertaken, specific actions need to be implemented to address the factors identified. Change management is an extensive topic in itself and beyond the remit of this article. Using the example indicated in Box 1, examples of some easy, actionable changes to mitigate increased waiting times include making the queueing policy more robust and implementing checks to ensure at least one wheelchair is available for patients when required. The process of risk management should not end with identifying risk, but with taking steps to reduce them for future care delivery.

\section{Education, training and CPD}

Healthcare and dentistry are fluid and rapidly changing. Techniques established even five years ago are now obsolete and therefore it is up to us as members of the clinical team to keep up with the latest innovations via continual education and training, which forms part of CPD. CPD is mandated by the General Dental Council (GDC). The GDC stipulates that registrants who are dentists undertake a minimum of 100 hours of verifiable CDP in a five-year cycle, with at least ten hours every two years. ${ }^{16} \mathrm{~A}$ list of recommended CPD as determined by the GDC can be seen in Box 2 .

It is important that CPD is informed by a personal development plan (PDP) which, as of 2018, became another GDC requirement. A PDP can 'help you to recognise educational needs as well as personal aspirations and will allow you to map out how you can achieve these. ${ }^{17}$ It is important when creating goals for yourself that they are 'SMART': specific, measurable, achievable, relevant and timebound. An example of a SMART objective can

\section{Box 1 Example of the 'Five whys' method of root cause analysis*}

'The patient's diagnosis of skin cancer was considerably delayed. Why?

The excision biopsy report was not seen by the surgeon. Why?

The report was filed in the patient's notes without being seen by the surgeon. Why?

It was the receptionist's job to do the filing. Why?

The junior doctors were busy with other tasks. Why?

The root cause is that the doctor's other tasks were seen as more important than filing. The system has now been changed. A copy of all biopsy reports are sent to the consultant surgeon responsible for the patient and no reports are filed unless they have been signed by a doctor.'

*Information derived from ${ }^{13}$

Fig. 2 Example of a root cause analysis using a fishbone diagram, reproduced with permission from NHS Improvement and ACT Academy, 'Cause and effect (fishbone)', 2018'4

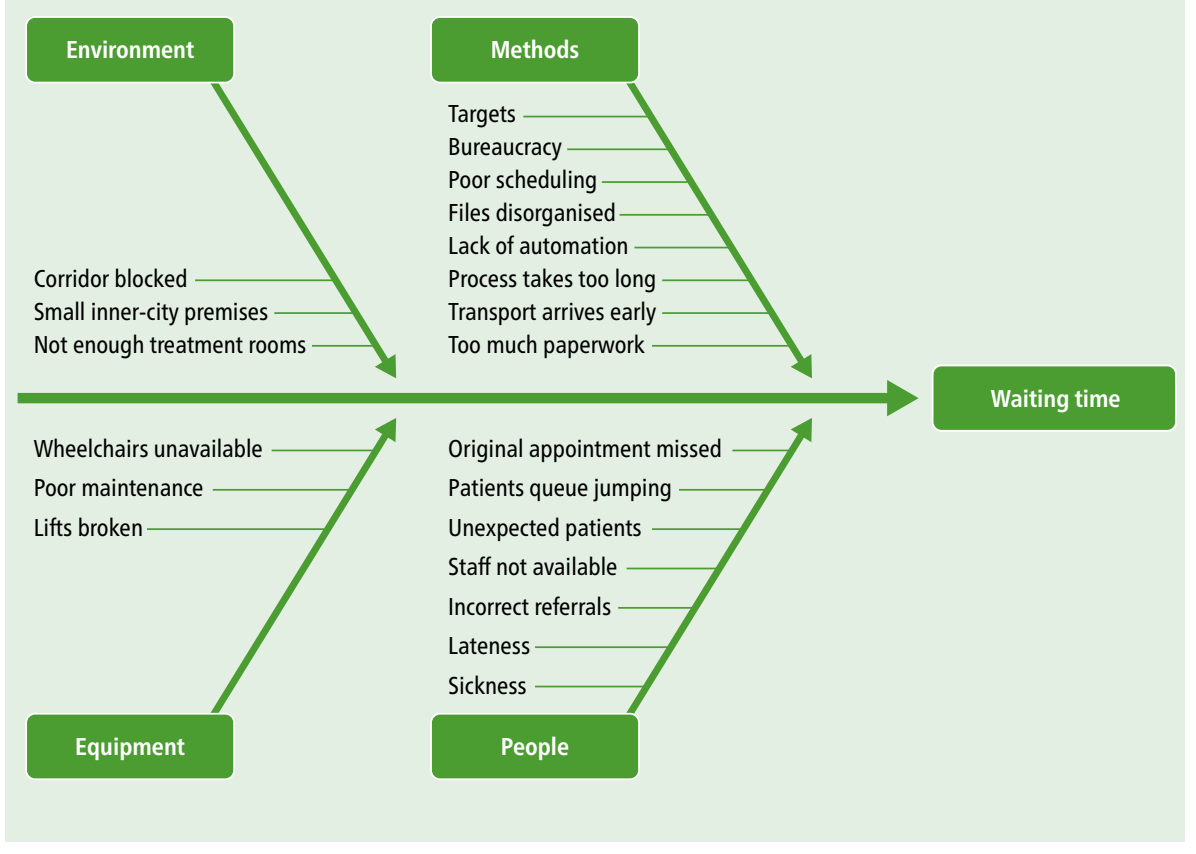

\section{Box 2 GDC-recommended CPD topics for dentists*}

Medical emergencies: at least ten hours per five-year CPD cycle with at least two hours per year

Disinfection and decontamination: at least five hours per CPD cycle

Radiography and radiation protection: at least five hours per CPD cycle

Legal and ethical issues

Complaints handling

Oral cancer: early detection

Safeguarding children and young adults

Safeguarding vulnerable adults.

*Information derived from ${ }^{20}$

be seen in Table 1. Once SMART objectives have been formulated, methods in which they can be actioned and recorded need to be set. Regular review and reflection on a PDP is important to ensure it meets these criteria and prioritises the next steps in learning. It can be all too easy to expend efforts towards learning tasks we don't necessarily want to develop in. Therefore, poorly planned training can have significant associated time and financial costs. Having focused goals will direct learning and allow practitioners to maximise their professional development, ultimately improving patient outcomes. 
Table 1 SMART objectives

\begin{tabular}{l|l} 
SMART objective & Description \\
\hline Specific & Specific to bitewing radiographs \\
\hline Measurable & At least 70\% of all radiographs \\
\hline Attainable/actionable & Actionable via clinical audit \\
\hline Realistic & Radiograph provision is realistic \\
\hline Timely & End of 2021 \\
\hline SMART objective & $\begin{array}{l}\text { By December 2021, I will ensure that at least 70\% of my bitewing } \\
\text { radiographs are rated as being of quality grade A. This will be determined } \\
\text { by a peer-conducted audit }\end{array}$ \\
\hline Not a SMART objective & $\begin{array}{l}\text { I want to be better at taking radiographs, specifically bitewing } \\
\text { radiographs this year }\end{array}$ \\
\hline
\end{tabular}

\section{Patient and public involvement}

The patients that we serve as dentists are the most important stakeholders in the care that we provide. It is therefore essential to ensure that their values and needs are taken into account when delivering their care. This can be difficult to implement, but looking at the local needs of the population is important in order to tailor the type of services we offer.

Readily attainable methods of patient and public involvement include conducting patient satisfaction questionnaires and collecting data on patient-reported outcome and experience measures. This provides us as healthcare professionals with direct feedback on aspects of our care which we are delivering well and may indicate areas for improvement. A national scheme undertaken to directly gain feedback from patients throughout primary and secondary care dental services was the Friends and Family Test (FFT). This was launched in 2003 and allows commissioners and service providers (including dentists) valuable in-action feedback on their care. ${ }^{18}$ Engagement from patients ultimately equates to an increase in responsiveness to clinical provision. ${ }^{19}$

\section{Staff and staff management}

Providing high-quality clinical care is a team endeavour, with the dentist assuming the role of leading the team and managing appropriately. This means having the right people in the right places at the right time to deliver the right care for the right patient. Staff need to be appropriately trained, motivated and willing to deliver the best possible care for their patients. In a dental practice setting, this may mean delivering staff training at regular intervals, in accordance with learning needs. This may be communication courses for reception staff or basic life support for the entire team.

Further methods of effectively managing the dental team include providing timely performance reviews to ensure all members of the team are progressing in relation to their PDP. If not, then action needs to be taken to CPD. Morning 'huddles' are frequently implemented in a hospital setting to ensure that the whole team is aware of any immediate challenges to their working day. Facilitating good communication among team members will foster better working relationships and ultimately allow for superior staff management.

\section{Information management}

As digital technology constantly improves, it takes on an ever-increasing role in our daily clinical practice. This brings with it certain issues that must be considered regarding the collection, management and use of patient in the form of directed tasks contributing information. There exist both legal and ethical obligations to ensure the secure storage of patient information. Patient information should also be used and shared judiciously with the appropriate justifications in their best interests. Practically, these measures are subject to constant change, reflecting the development in present technologies, requiring regular re-evaluation and update of information systems.

The General Data Protection Regulation (GDPR) is a European Union law introduced in 2018 which governs the way organisations, including hospital and dental healthcare systems, utilise, store, manage and share personal information. The law promotes data privacy and security in a time where more individuals are more forthcoming with sharing their personal information. There are specific data protection principles which are outlined in Article 5 of the GDPR, which can be seen in Table $2 .{ }^{21}$ Valid, informed consent must be gained by patients to process their personal data for specified outcomes. Such consent can be withdrawn at any time. Processing, or sharing, personal data can be done for the following reasons: ${ }^{22}$

1. When the patient has given you their valid, informed consent to do so (for example, signing up to practice marketing e-mails)

2. Personal data processing is required to enter into contract with the patient (for example, using a practice finance package to aid with treatment costs)

3. Complying with legal obligations (for example, court order mandate)

4. To save a patient's or an individual's life

5. To perform a task in the public interest or to perform an official function (for example, disclosing to official bodies that a poorly
Table 2 GDPR data protection principles ${ }^{20}$

\begin{tabular}{l|l} 
Principle & Explanation \\
\hline $\begin{array}{l}\text { Lawfulness, fairness and } \\
\text { transparency }\end{array}$ & Processing of personal data should be lawful, fair and transparent \\
\hline Purpose limitation & $\begin{array}{l}\text { Personal data collected should only be utilised for the specified reason as to why } \\
\text { it was initially collected }\end{array}$ \\
\hline Data minimisation & $\begin{array}{l}\text { The absolute minimum data should be collected and processed for the desired } \\
\text { purpose }\end{array}$ \\
\hline Accuracy & $\begin{array}{l}\text { Personal data should be up to date. Inaccurate data should be erased or rectified } \\
\text { without delay }\end{array}$ \\
\hline Storage limitation & $\begin{array}{l}\text { Personal data should only be stored for as long as needed. This will be dictated by } \\
\text { the specified reason as to why said data was initially collected }\end{array}$ \\
\hline $\begin{array}{l}\text { Integrity and } \\
\text { confidentiality }\end{array}$ & $\begin{array}{l}\text { Processing to ensure security of personal data. This includes processing of data } \\
\text { not consented for, loss or damage of personal data, and sharing of data to third } \\
\text { parties }\end{array}$ \\
\hline Accountability & $\begin{array}{l}\text { A data 'controller' should be able to demonstrate compliance with all of the } \\
\text { principles highlighted above }\end{array}$ \\
\hline
\end{tabular}


controlled epileptic patient has driven to your clinic appointment)

6. There is a legitimate interest to process a patient's personal data (for example, referring a patient to secondary care services).

It is important that all healthcare professionals and organisations alike are familiar with the new legislation and their associated nuances.

\section{Delivering effective clinical governance}

\section{Beams, not pillars}

It is important to note that the various 'pillars' that comprise clinical governance do not stand alone. Perhaps a better metaphor would be to describe them as intersecting beams, all supporting one another. Ultimately, the different aspects of clinical governance are interdependent, where one cannot be delivered without the other. For example, the SMART objective example given in Figure 2 touches on aspects of CPD, clinical audit, and staff and staff management. Furthermore, the set standards are evidence-based, which is yet another beam under the clinical governance umbrella. Therefore, to deliver clinical governance coherently, a culture needs to be built up within an institution. This institution can be just as much a primary care practice as an NHS foundation trust.

\section{Role of leadership in clinical governance}

In order to promote such a culture, there needs to be effective leadership to ensure the message supported by the term clinical governance permeates through all layers of any given organisation. There are differing leadership styles, with Goleman et al. ${ }^{23}$ proposing six types: affiliative, authoritative, coaching, democratic, pacesetting and coercive. There is no better type of leadership style, but a good leader should be able to adopt differing styles based on the needs of their team. Good leadership not only ensures aspects of clinical governance are met, but also facilitates better patient outcomes and increased workforce motivation. ${ }^{24}$ Within a dental practice, such leadership is often provided by the principal dentist or practice manager, ensuring that an environment reflective of the clinical excellence is promoted. In a hospital setting, there is a well-structured hierarchy including a clinical governance lead for each department and a member of the executive committee responsible for the clinical governance of the trust.

Regardless of the clinical setting, effective leadership needs to be in place to ensure all employees in patient-facing and nonpatient-facing roles are on board with clinical governance requirements. Clear goals should be set to ensure that all members of staff are working synergistically to realise them, with aspects of clinical governance at the forefront of their minds.

\section{Concluding remarks}

Clinical governance is a broad area; this article has just touched on its basic principles and what they entail. Despite this, aspects of this 'umbrella term' dictate what we do as dentists on a daily basis. With a greater knowledge of what this involves, the hope is that our delivery of care to our patients improves and we evolve to become better clinicians. This paper has introduced methods in which practitioners can approach clinical tasks and audits, and mitigate adverse incidents in the future. As dentists, we lead our clinical teams and we must bear the responsibility of promoting clinical governance to ensure that others follow our example. This will foster a safer, more effective clinical environment which will safeguard and promote a high quality of care for our deserving patients.

\section{References}

1. Care Quality Commission. Nigel's surgery 65: Effective clinical governance arrangements in GP practices. 2017. Available at https://www.cqc.org.uk/guidanceproviders/gps/nigels-surgery-65-effective-clinicalgovernance-arrangements-gp-practices (accessed July 2020).

2. Scally G, Donaldson L J. Looking forward: clinical governance and the drive for quality improvement in the new NHS England. BMJ 1998; 317: 61-65.

3. Walshaw E, Mannion C J. Dentists are humans tooeducation in human factors within dental care. Br Dent J 2018; 224: 901-904.

4. American Dental Association. Policy on Evidence-Based Dentistry. 2013. Available at https://www.ada.org/en/ about-the-ada/ada-positions-policies-and-statements/ policy-on-evidence-based-dentistry (accessed July 2020).

5. Patel K, Pajpani M. Making sense of the literature: An introduction to critical appraisal for the primary care practitioner. Br Dent J 2020; 229: 551-555.

6. SDCEP: Management of acute dental problems during COVID-19 pandemic. 2020. Available at https://www. sdcep.org.uk/wp-content/uploads/2020/03/SDCEPMADP-COVID-19-guide-300320.pdf (accessed July 2020).

7. FGDP(UK). Ideas for audit - developing local guidelines 2019. Available at https://www.fgdp.org.uk/selectioncriteria-dental-radiography/92-ideas-audit--developing-local-guidelines (accessed July 2020).

8. World Health Organisation. WHO surgical safety checklist. 2020. Available online at https://www.who. int/patientsafety/safesurgery/checklist/en/ (accessed July 2020).

9. Care Quality Commission. Regulation 20: Duty of Candour. 2020. Available at https://www.cqc.org. uk/guidance-providers/regulations-enforcement/ regulation-20-duty-candour\#guidance (accessed August 2020).

10. British Orthodontic Society. Adverse incidence reporting. 2014. Available at https://www.bos.org.uk/ Professionals-Members/Adverse-Incident-Reporting (accessed July 2020).

11. National Patient Safety Foundation: RCA Improving root causes analyses and actions to prevent harm. 2015. Available at https://www.ashp.org/-/media/ assets/policy-guidelines/docs/endorsed-documents/ endorsed-documents-improving-root-cause-analysesactions-prevent-harm.ashx (accessed July 2020).

12. Rooney J J, VendenHeuvel L N. Root cause analysis for beginners. Qual Proc 2004; 37: 45-53.

13. NHS Improvement. Root cause analysis using five whys. Available at https://improvement.nhs.uk/ documents/2156/root-cause-analysis-five-whys.pdf (accessed July 2020).

14. NHS Improvement. Cause and effect. Available at https://improvement.nhs.uk/documents/2093/causeeffect-fishbone.pdf (accessed July 2020).

15. NHS Improvement. Brainstorming. Available at https:// improvement.nhs.uk/documents/2091/brainstorming. pdf (accessed July 2020).

16. General Dental Council. Enhanced CPD guidance. 2018 Available at https://www.gdc-uk.org/docs/defaultsource/enhanced-cpd-scheme-2018/enhanced-cpdguidance-for-professionals.pdf (accessed July 2020).

17. Rimmer A. How do I prepare a personal development plan? BMJ 2018; DOI: 10.1136/bmj.k4725.

18. NHS. Friends and Family Test (FFT). 2020. Available at https://www.nhs.uk/using-the-nhs/about-the-nhs/ friends-and-family-test-fft/ (accessed July 2020).

19. Halligan A, Donaldson L. Implementing clinical governance: turning vision into reality. BMJ 2001; 322: 1413-1417.

20. General Dental Council. Recommended CPD topics. 2019. Available at https://www.gdc-uk.org/education$\mathrm{cpd} / \mathrm{cpd}$ /recommended-cpd-topics (accessed July 2020).

21. GDPR.EU. Article 5 GDPR: Principles relating to processing of personal data. Available at https://gdpr. eu/article-5-how-to-process-personal-data/ (accessed August 2020).

22. GDPR.EU. Article 6 GDPR: Lawfulness of processing. Available at https://gdpr.eu/article-6-how-to-processpersonal-data-legally/ (accessed August 2020).

23. Goleman D, Boyatzis R E, McKee A. The new leaders: Transforming the art of leadership into the science of results. London: Time Warner Paperbacks, 2003.

24. Grocock R. Leadership in dentistry. Br Dent J 2020; 228: 882-885. 